\title{
Experimental Review of Baryons in the Nuclear Medium
}

\section{S. Schadmand ${ }^{a *}$}

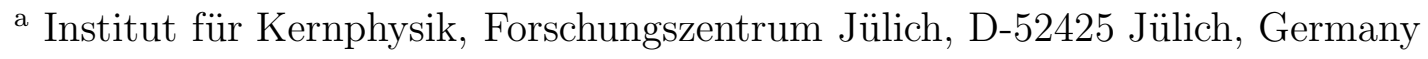

Inclusive studies of nuclear photoabsorption have provided clear evidence of medium modifications but the results have not yet been explained in a model independent way. A deeper understanding of the situation is anticipated from a detailed experimental study of meson photoproduction from nuclei in exclusive reactions. Recent results on meson production in photonuclear experiments indicate a large difference between quasifree meson production from the nuclear surface and non-quasifree components.

\section{INTRODUCTION}

Photoabsorption experiments on the free nucleon demonstrate the complex structure of the nucleon and its excitation spectrum. The lowest resonance is called $\Delta(1232)$ which is

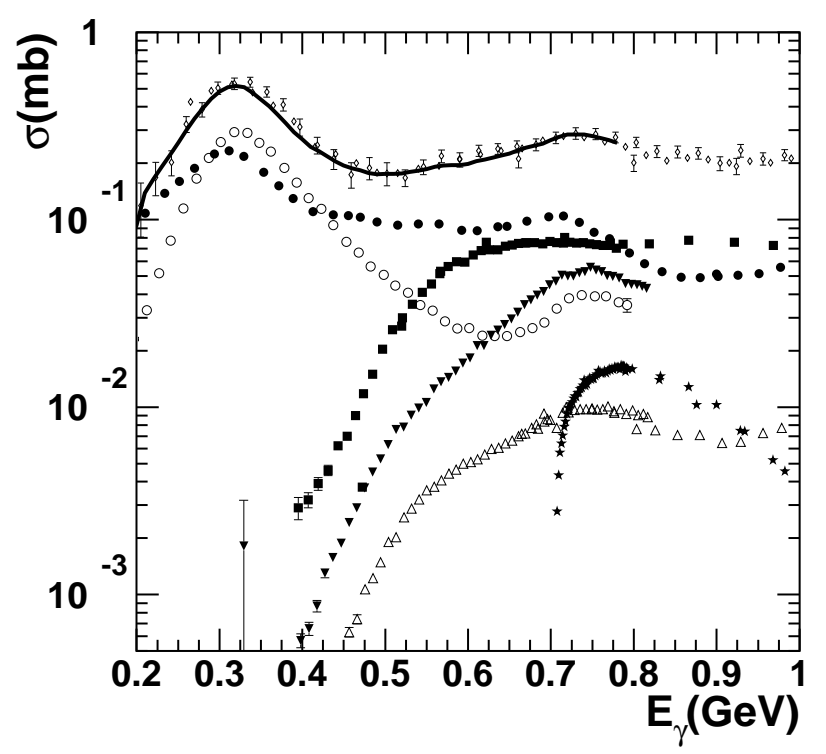

Figure 1. Photoabsorption cross section on the proton and decomposition into meson production channels. Small open circles are the photoabsorption data compilation from [1]. The experimental meson production cross sections are: single $\pi^{+}$(solid circles) from [23, single $\pi^{\circ}$ (open circles) from [4, $\pi^{+} \pi^{-}$ (solid squares) from [5]6], $\pi^{+} \pi^{\circ}$ (downward solid triangles) from [7], $\pi^{\circ} \pi^{\circ}$ (upward open triangles) from [89], and $\eta$ (stars) from [1011]. The solid line is the sum of the meson channels up to $800 \mathrm{MeV}$.

a $\mathrm{P}_{33}$ state in the common notation $\left(\mathrm{L}_{(2 I)(2 J)}\right)$ with a pole mass of $1232 \mathrm{MeV}$. It is prominently excited by incident photons of $0.2-0.5 \mathrm{GeV}$. The following group of resonances, $\mathrm{P}_{11}(1440), \mathrm{D}_{13}(1520)$, and $\mathrm{S}_{11}(1535)$, is called the second resonance region $\left(\mathrm{E}_{\gamma}=0.5\right.$ $0.9 \mathrm{GeV})$. The observed resonance structures have been studied using their decay via

\footnotetext{
${ }^{*}$ Work supported by Deutsche Forschungsgemeinschaft, UK Science and Engineering Research Council,
} and Schweizerischer Nationalfonds. 
light mesons, showing that the photoabsorption spectrum can be explained by the sum of $\pi, \pi \pi$ and $\eta$ production cross sections. Fig. 1 shows the photoabsorption cross section on the proton along with the experimental meson photoproduction cross sections. The shapes of the meson cross sections reflect the resonance structures observed in photoabsorption showing that the mesons are mostly decay products of the respective resonances. Single pion production is dominant in the region of the $\Delta(1232)$ resonance. Also, the three resonances comprising the second resonance region, decay to $\sim 50 \%$ via single pion emission. This fact has been extensively exploited in partial wave analyses. Above $\mathrm{E}_{\gamma} \approx 0.4 \mathrm{GeV}$, the photoproduction of two pions is kinematically possible and single $\pi$ production looses in dominance. The solid line in Fig. 1 represents the sum of the meson cross sections up to $0.8 \mathrm{GeV}$ and demonstrates that the photoabsorption cross section on the proton can be explained by its decomposition into meson production.

The $\eta$ production threshold is located at $\mathrm{E}_{\gamma} \approx 700 \mathrm{MeV}$. The steeply rising $\eta$ cross section in Fig. 11 is characteristic for an s-wave resonance. The angular distributions of the $\eta$ emission are consistent with this observation and the cross section peaks around the mass pole of the $S_{11}(1535)$ resonance [10 11]. This resonance is unique in the sense that is has a strong decay branch of $30-55 \%$ into $\eta$ mesons. Thus, $\eta$ production is considered characteristic for the $\mathrm{S}_{11}(1535)$ resonance. Above the $\eta$ threshold, the cross section basically displays the resonance line shape enabling detailed studies of that state. However, the contribution to the total is small.
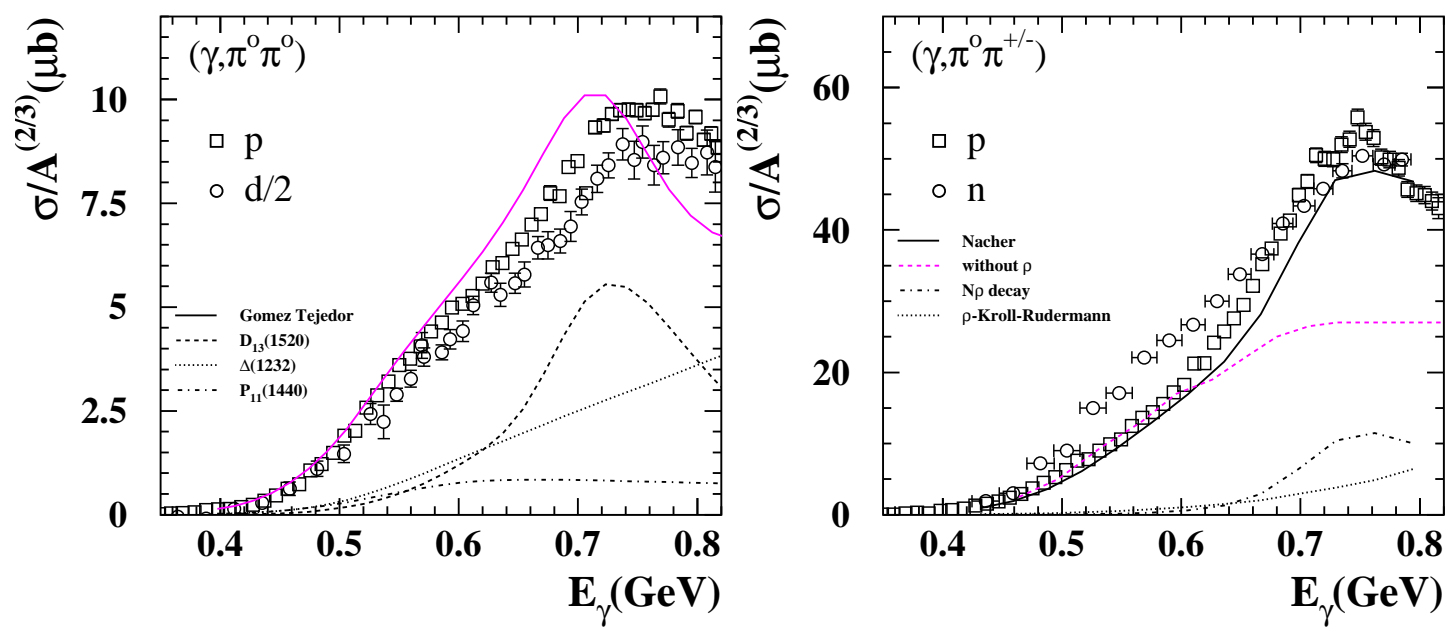

Figure 2. Double pion production from the nucleon. The curves are calculations from 1213 . Left: comparison of $2 \pi^{\circ}$ from the proton and deuteron [14. Right: comparison of $n \pi^{\circ} \pi^{+}$[5] with $p \pi^{\circ} \pi^{-}$[15].

In the second resonance region, the dominant contribution comes from the $\mathrm{D}_{13}(1520)$ resonance which has the strongest coupling to the incident photon. Single as well as double pion production channels display structure at the corresponding resonance mass, i.e. around $\mathrm{E}_{\gamma} \approx 760 \mathrm{MeV}$. Fig 2 shows results on the photoproduction of pion pairs 
on the nucleon. As could already be seen from Fig. 1, the meson production channels involving charged pions are dominant as expected in electromagnetic excitation processes. On the proton, three isospin combinations of pion pairs can be produced. $\pi^{\circ} \pi^{\circ}$ and $\pi^{+} \pi^{-}$production revealed that the resonance decays sequentially via an intermediate $\Delta$ state [516. In $\pi^{+} \pi^{\circ}$ the same behavior was found. In addition, a decay branch of $20 \%$ $N^{\star} \rightarrow N \rho$ was deduced [713]. On account of its dominance, the $\mathrm{D}_{13}(1520)$ resonance is said to be tagged by double pion production. However, calculations by Gomez Tejedor and Oset [12], showed that the $N^{*}$ contribution to double pion photoproduction by itself is not large but rather stems from an interference with other terms.

\section{NUCLEAR PHOTOABSORPTION}

The left panel of Fig. 3 shows the nuclear photoabsorption cross section per nucleon as an average over the nuclear systematics [17].
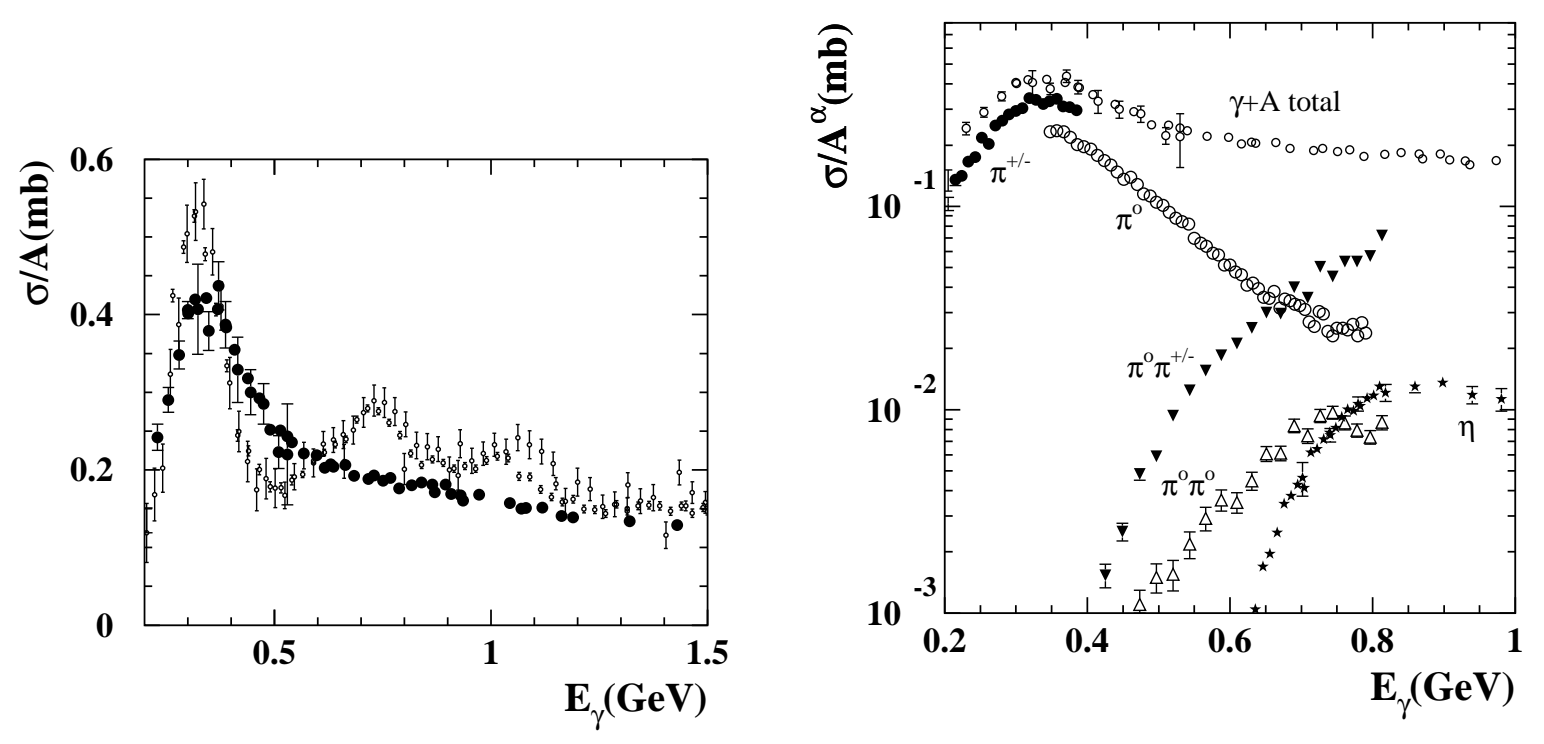

Figure 3. Left: Nuclear photoabsorption cross section per nucleon as an average over the nuclear systematics [17] (full symbols) compared to the absorption on the proton [1] (open symbols). Right: Status of the decomposition of nuclear photoabsorption into meson production channels (scaled with $\mathrm{A}^{\alpha}, \alpha=2 / 3$ ). Small open circles are the average nuclear photoabsorption cross section per nucleon $(\alpha=1)$ [17]. Meson production data are from 1819202122 .

The $\Delta$ resonance is broadened and slightly shifted while the second and higher resonance regions seem to have disappeared. The right panel shows the status of the decomposition of nuclear photoabsorption into meson production channels. The available experimental meson cross sections are exclusive measurements, investigating quasifree production. It 
can be inferred from the figure that the sum of the cross sections, which are missing the purely charged final states, cannot reproduce the shape of the total photoabsorption from nuclei.

Mosel et al. 23, have argued that an in-medium broadening of the $\mathrm{D}_{13}(1520)$ resonance is a likely cause of the suppressed photoabsorption cross section. The calculation is based on the BUU equation which describes the space time evolution of the spectral phase space density of an ensemble of interacting particles. For the baryons, the mean field potential is determined as described in 24. Here, nuclear incompressibilities corresponding to a hard equation of state (momentum independent potential) and a medium equation of state (momentum dependent potential) are employed. The photon-nucleus reaction is modelled in terms of the absorption of the photon on a single nucleon (quasifree process). Over a more comprehensive energy range, this leads to final states $\mathrm{P}_{33}(1232), \mathrm{D}_{13}(1520)$, $\mathrm{S}_{11}(1535), \mathrm{F}_{15}(1680), N \pi, N \pi \pi, N V, K \Lambda, K \Sigma$ and $K \bar{K} N$. The states are prepared according to the respective cross sections. Final state interactions are described by a set of BUU equations. The cross sections for the $\gamma A$ reaction are determined by averaging over an ensemble of such elementary reactions as outlined in [25]. Besides Fermi motion, binding effects and Pauli blocking, collisional broadening of the most important resonances $\mathrm{P}_{33}(1232), \mathrm{D}_{13}(1535)$ and $\mathrm{S}_{11}(1535)$ is accounted for. The left panel of Fig. 4 shows the total photonuclear cross sections where different scenarios are presented. The use of a momentum dependent $N^{*}$ potential leads to a smearing of the $\mathrm{D}_{13}(1520)$ because the momentum dependence leads to a shift of the effective mass. For a $\mathrm{D}_{13}(1520)$ produced with momenta around $800 \mathrm{MeV}$, the nucleon potential almost vanishes. This shift amounts to $\Delta m^{*} \approx 50 \mathrm{MeV}$. The broadening is due to the strong increase of the width with the mass. An almost complete disappearance of the resonant structure is observed but the experimental data are still overestimated. Altering the resonant contribution by using an enhanced in-medium width leads to a more smeared and reduced cross section. An enhancement of the $N \rho$ width at nuclear matter density by about a factor 10 was found with a total width at the pole mass of about $335 \mathrm{MeV}$. The use of this in-medium $N \rho$ width includes the full mass, momentum, and density dependence and leads to the solid curve in Fig. 4. The description of the experimental data is considerably improved. However, a bump structure survives for photon energies around $650 \mathrm{MeV}$ which is caused by the strong mass dependence of the $\mathrm{D}_{13}$ width which is reflected in the in-medium width.

Hirata et al. 26] have argued that a change of the interference effects in the nuclear medium is one of the most important reasons for the suppression of the resonance structure. The right panel of Fig. 4 shows the calculated cross section per nucleon. The contributions of one pion and two pion production, many body absorption through the $\Delta$-nucleus and $N^{*}$-nucleus state, as well as many-body absorption through the $\pi \Delta$-nucleus state are shown by themselves. Here, two-pion photoproduction is about 3 times smaller than in the elementary process due to cooperative effects between the different medium effects as in spreading potentials for $\Delta$ and $N^{*}$, pion distortion, and modified interferences among the related reaction processes. The cross sections of the other many-body processes are almost flat in the energy range above $600 \mathrm{MeV}$ and small. The excitation peak around the position of the $N^{*}$ resonance in the total nuclear photoabsorption cross section is indeed not present in the calculation. However, the model underestimates the nuclear cross section in the valley region between 380 and $500 \mathrm{MeV}$ by about 15 percent. 

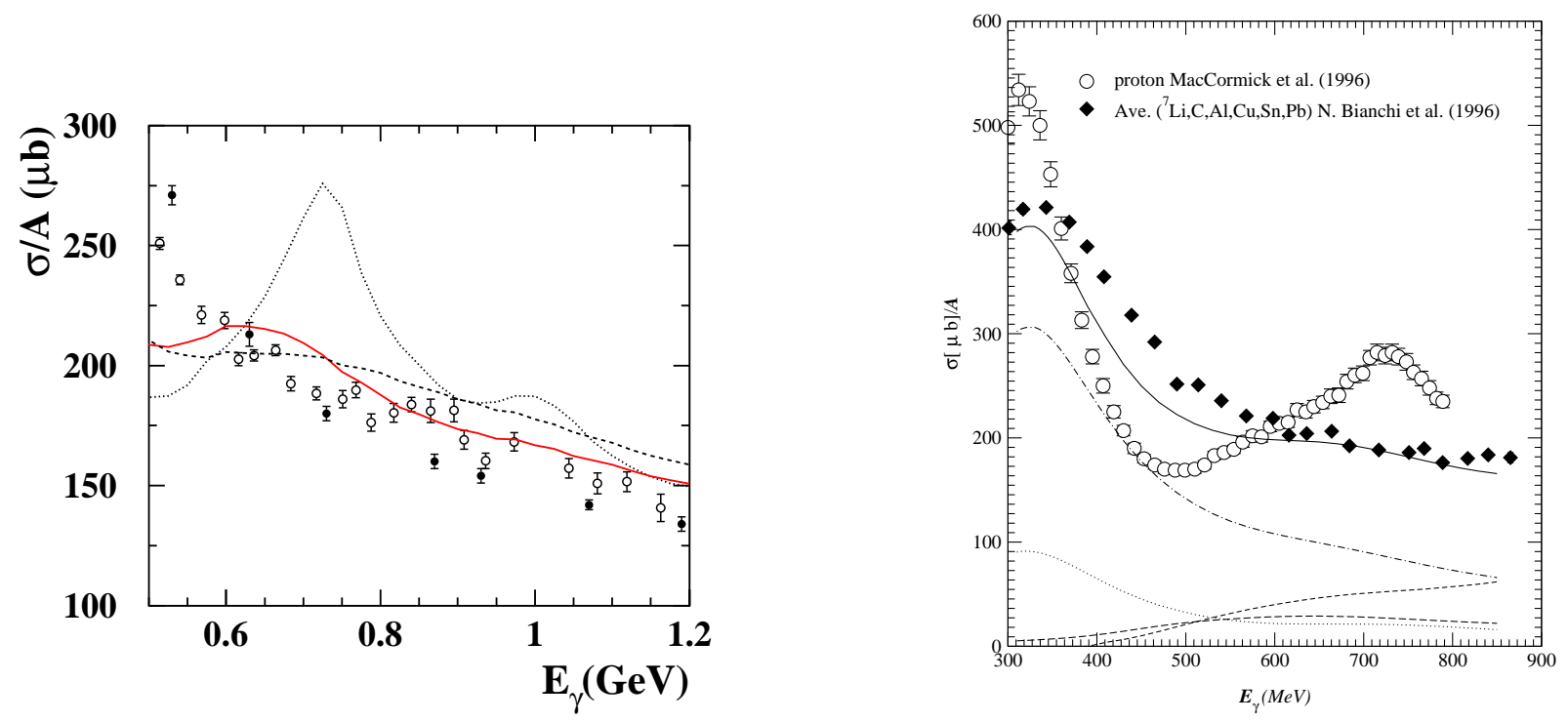

Figure 4. Left: Modification of the second resonance region for the $\gamma^{* 40} \mathrm{Ca} \rightarrow X$ cross section from BUU calculations [24]. The solid curve is calculated with an explicit enhancement of the $N \rho$ width, the dashed curve with a momentum dependent potential. Dotted curve: photoabsorption on the free proton. Experimental data are from [27]. Right: Total nuclear photoabsorption cross section on nuclei according to Hirata et al. [26. The solid curve is the full calculation. Individual contributions of the absorption processes are shown by themselves: one pion production (dash-dotted line), two pion production (dashed line), many body absorption through the $\Delta$-nucleus state and $N^{*}$ nucleus state (dotted line), and many body absorption through the $\pi \Delta$-nucleus state (long dashed line).

It is inferred that there must be an important processes enhancing nuclear photoabsorption in the valley region. Here, the intermediate pion and $\rho$ meson are far off-shell and two nucleons could explicitly contribute. In addition, the cross sections are underestimated slightly at the $\Delta$ resonance energy around $320 \mathrm{MeV}$ as coherent $\pi^{0}$ production is not included in the calculation.

It may be concluded that inclusive reactions like total photoabsorption do not allow a detailed investigation of in-medium effects. A deeper understanding of the situation is anticipated from the experimental study of meson photoproduction on nucleons embedded in nuclei in comparison to studies on the free nucleon.

\section{MESON PHOTOPRODUCTION FROM NUCLEI}

In reactions from the free nucleon, baryon resonance properties are extracted by tagging on their characteristic meson decay. The same procedure may be applied to nucleon excitations in the nuclear medium. In the second resonance region, double pion production aims at the resonances $\mathrm{D}_{13}(1520)$ and $\mathrm{P}_{11}(1440)$ while $\eta$ production is characteristic for the $\mathrm{S}_{11}(1535)$ resonance. As pointed out above, the three resonances in the second resonance 
region decay to roughly $50 \%$ via single pion emission.

The most trivial medium modification is the broadening of the excitation functions due to Fermi motion. The decay of the resonances is further modified by Pauli-blocking of final states, which reduces the resonance widths. In addition, decay channels like $\mathrm{N}^{\star} \mathrm{N} \rightarrow \mathrm{NN}$ cause collisional broadening. Both effects cancel to some extent and it is a priori not clear which will dominate.
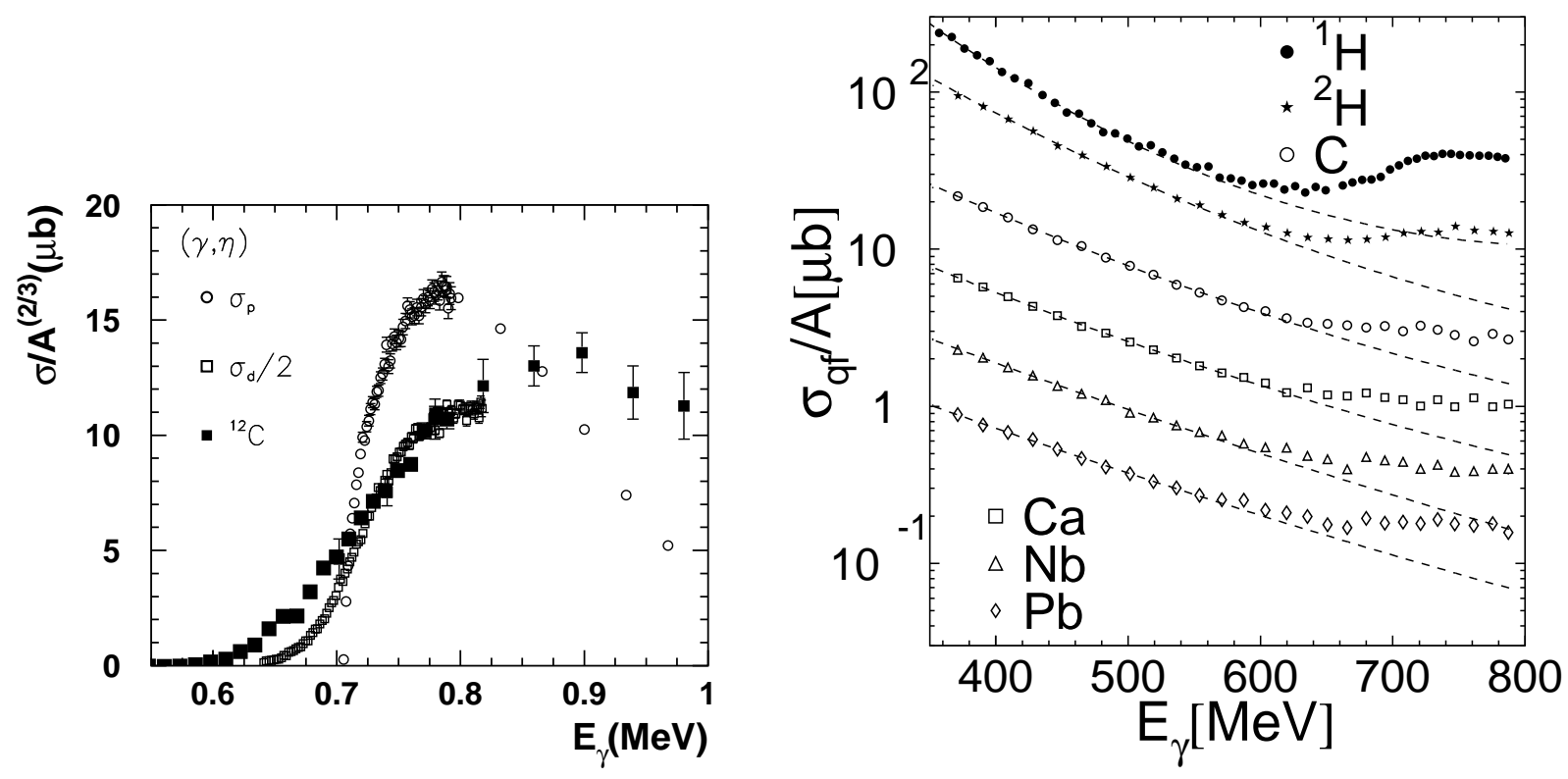

Figure 5. Left: Comparison the total cross section per nucleon for $\eta$ photoproduction on nucleons [1011] and using a carbon target 2021]. Right: Total cross section per nucleon for single $\pi^{\circ}$ photoproduction in the second resonance region for the nucleon and for nuclei. The scale corresponds to the proton data, the other data are scaled down by factors $2,4,8,16,32$, respectively. The dashed curves are fits to the data in the energy range $350-550 \mathrm{MeV}$.

\section{1. $\eta$ PRODUCTION}

On the free proton, the photoproduction of $\eta$-mesons in the second resonance region proceeds almost entirely through the excitation of the $\mathrm{S}_{11}(1535)$ resonance. An observation of the reaction over a series of nuclei 2021] did not show a depletion of the in-medium strength. The left panel of Fig. 5 compares the total cross section per nucleon for $\eta$ photoproduction on the proton and using a carbon target. This result is in line with theoretical findings that the change of the $S_{11}$ self energy in the medium is small [2829] and are in agreement with model calculations that take the trivial in-medium effects and final state interactions into account 2530. A recent study shows that the data could be described over the full energy range by applying a momentum dependent $S_{11}$ potential [31. 


\subsection{SINGLE $\pi^{\circ}$ PRODUCTION}

An attempt to study the in-medium properties of the $\mathrm{D}_{13}$ resonance was undertaken with a measurement of quasifree single $\pi^{\circ}$ photoproduction [19] which, on the free nucleon, is almost exclusively sensitive to the $\mathrm{D}_{13}$ resonance. The right panel of Fig. 5 summarizes the results. Strong quenching of the $\mathrm{D}_{13}$-resonance structure is found for the deuteron with respect to the nculeon. However, an indication of a broadening or a suppression of the $\mathrm{D}_{13}$ structure in heavy nuclei is not observed. Model predictions agree with the pion photoproduction data only under the assumption of a strong broadening of the resonance, other effects seem to be missing in the models. This casts doubt on the interpretation of the total photoabsorption data via resonance broadening. In contrast to the case of total photoabsorption, the second resonance bump remains visible. However, exclusive reaction channels are dominated by the nuclear surface region where in-medium effects are smaller. Furthermore, as discussed in [32], resonance broadening effects are even more diluted for reactions which do not contribute to the broadening, due to the averaging over the nuclear volume.

\subsection{DOUBLE PION PRODUCTION}
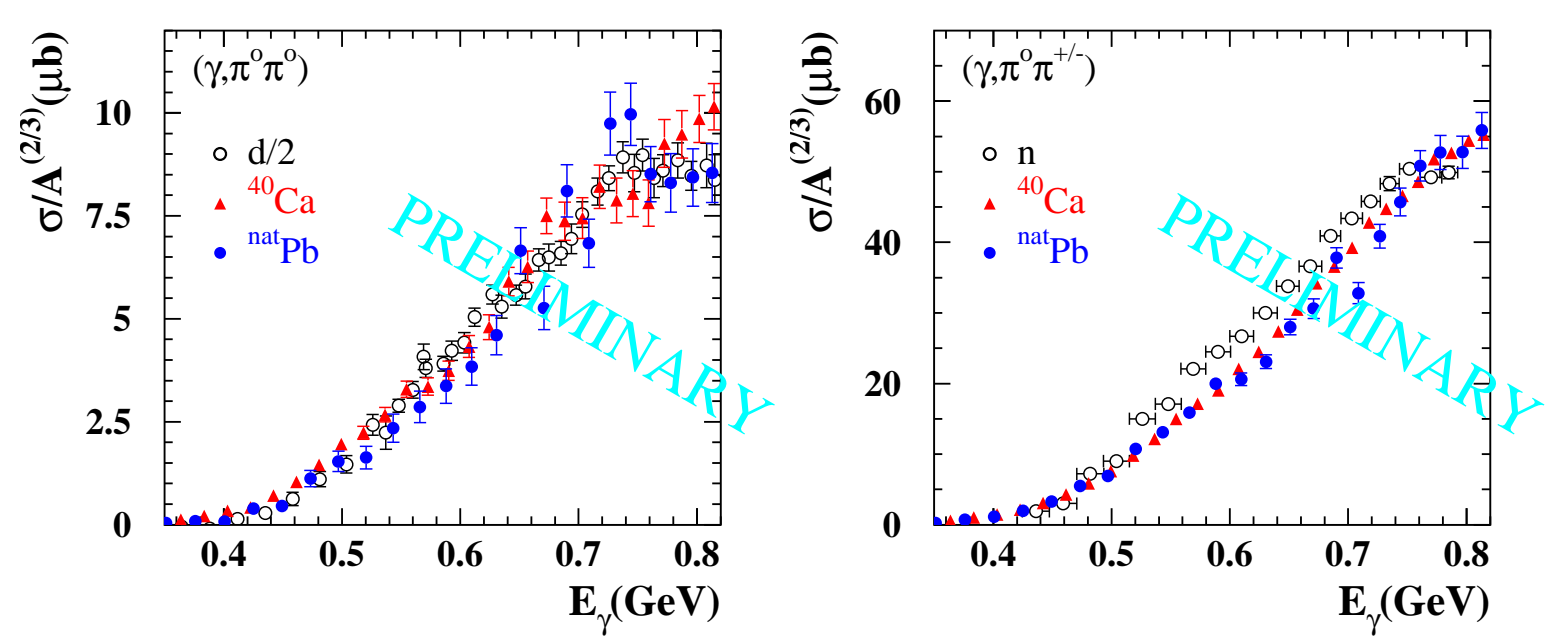

Figure 6. Preliminary total cross sections for $\pi \pi$ photoproduction from lead [22] along with results from the deuteron 1533 . The nuclear cross sections are divided by $\mathrm{A}^{2 / 3}$, the $\pi^{\circ} \pi^{\circ}$ deuteron cross section by 2 .

Fig. 6] shows preliminary cross sections for $\pi^{\circ} \pi^{\circ}$ and $\pi^{\circ} \pi^{ \pm}$photoproduction on calcium and lead from a recent TAPS analysis. The nuclear cross sections are divided by $\mathrm{A}^{2 / 3}$ and compared to results from the free proton and from nucleons bound in deuterons. With the scaling with $\mathrm{A}^{2 / 3}$, the nuclear data agree almost exactly with the cross sections on the nucleon. Thus, the total nuclear $\pi \pi$ cross sections do not seem to show any modification beyond absorption effects. It may be speculated that the strong $2 \pi$ decay branch via $\Delta$ intermediate states $\left(N^{\star} \rightarrow \Delta \pi \rightarrow N \pi \pi\right)$, together with the fact that the $\Delta$ 
resonance itself does not dramatically change in medium, dominate this behavior. Also, in the reaction $\pi^{\circ} \pi^{ \pm}$, the two pions can stem from the decay of the $\rho$ meson while the decay $\rho \rightarrow \pi^{\circ} \pi^{\circ}$ is forbidden. Accordingly, detailed studies of differential cross sections might reveal different modifications of the $\pi \pi$ correlations. A first result came from the investigation of $\pi \pi$ invariant mass distributions in the incident photon energy range of 400-460 MeV [34] providing indication of an effect consistent with a significant in-medium modification in the $A\left(\gamma, \pi^{\circ} \pi^{\circ}\right)(I=J=0)$ channel.

\subsection{SUMMARY}

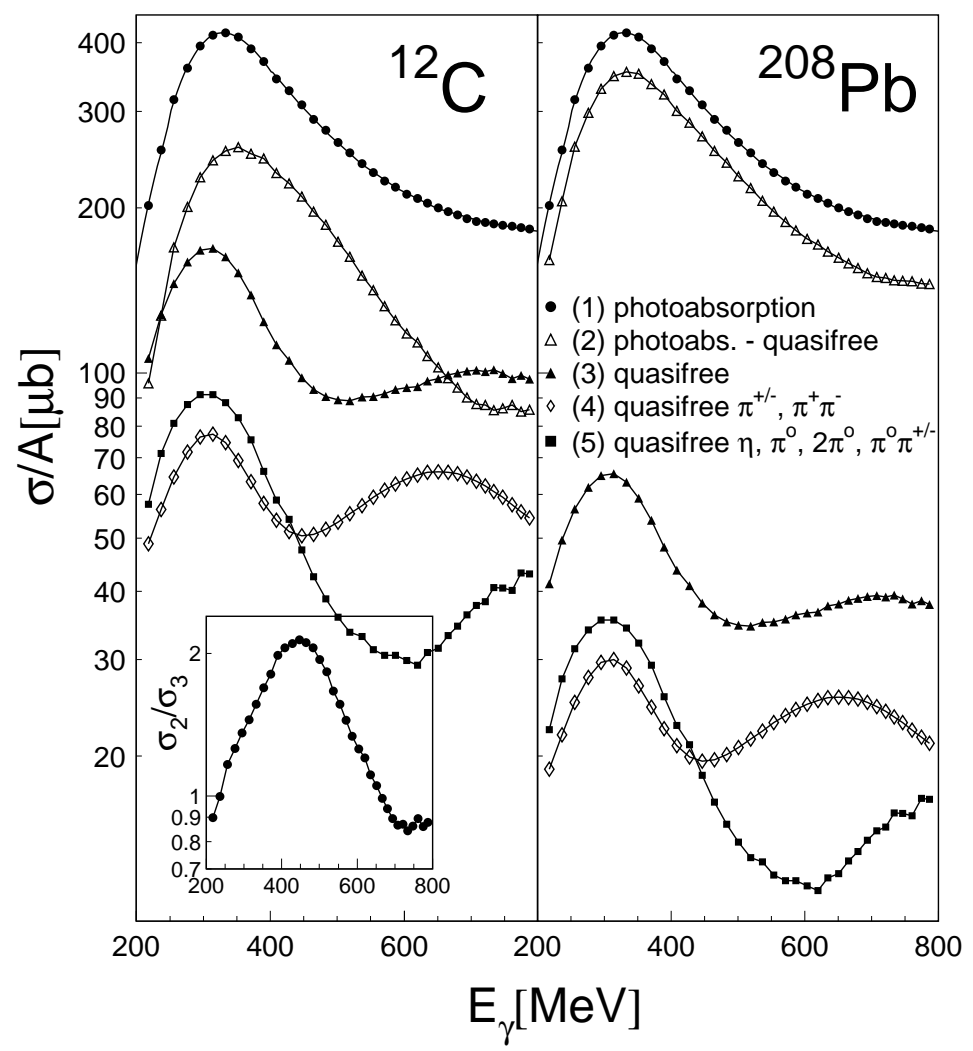

Figure 7. Decomposition of the total photoabsorption cross section (1) for carbon (left) and lead (right) 35. The sum of quasifree cross sections (3) is built from an approximation of purely charged final meson states (4) and the available cross sections containing at least one neutral meson (5). Curve (2) represents the difference of the estimated total quasifree meson production from the total absorption cross section. Inset: ratio of (2) and (3) for carbon.

The information about meson photoproduction from nuclei was obtained with the photon spectrometer TAPS in combination with the Glasgow tagged photon facility at MAMIB in Mainz. Technically, the experimental setup was tuned towards the detection of neutral mesons and charged pions could only be reliably detected in the presence of at least 
one photon (thus, in the presence of a $\pi^{\circ}$ ), from relative time-of-flight information. Data on the photoproduction of purely charged meson final states from nuclei are not available for the second resonance region. However, the neutral quasifree reactions consistently follow the scaling with the nuclear surface and charged pions will undergo similar final state effects. Thus, it is reasonable to assume the same scaling behavior and the quasifree cross section for charged meson production from the deuteron cross section has been approximated in Ref. 35]. The deuteron cross section was folded with a typical momentum distribution for nuclei in order to account for the stronger Fermi motion effects.

The result for carbon is shown in Fig. [7(left side, curve (4)) together with the quasifree cross section for neutral and mixed charged states (curve (5)), and the total photoabsorption cross section (curve (1)). The behavior for heavier nuclei is qualitatively the same (see Fig. 17, right side). Here, the all cross sections are scaled by $A$ and the inherent $A^{2 / 3}$ scaling of the quasifree reactions make the latter relatively less important for heavier nuclei. The sum of the quasifree meson production cross sections (curve (3)) shows clear signs of the $\Delta$ resonance and the second resonance region. The flattening is mainly due to Fermi motion effects. The excitation function reflects the typical response of the low density nuclear surface regions to photons. The difference between this cross section and total photoabsorption represents the typical response of the nuclear volume (curve(2)) where isolated resonance peaks are not seen. The inset in the figure shows the ratio of these two excitation functions for carbon. The most striking feature is the buildup of strength at incident photon energies around $400 \mathrm{MeV}$ in the volume component as compared to the quasifree surface reactions. It is known that two-body absorption mechanisms like $\gamma \mathrm{NN} \rightarrow N \Delta$ are non-negligible in this energy range [36, but it is not known if they can explain the effect. Further progress in the models is necessary for an understanding of this behavior. Also, it would be desirable to complete the experimental picture by investigating single charged pion as well as $\pi^{+} \pi^{-}$production from nuclei.

\section{CONCLUSIONS}

The systematic study of total cross sections for single $\pi^{\circ}, \eta$, and $\pi \pi$ production over a series of nuclei has not provided an obvious hint for a depletion of resonance yield. The observed reduction and change of shape in the second resonance region are mostly as expected from absorption effects, Fermi smearing and Pauli blocking, and collisional broadening. The sum of experimental meson cross sections for neutral and mixed charged states between 400 and $800 \mathrm{MeV}$ demonstrates the persistence of the second resonance bump when at least one neutral meson is observed.

The current results indicate large differences between quasifree meson production from the nuclear surface and non-quasifree components. The quasifree part does not show a suppression of the resonance structures in the second resonance region. However, resonance structures seem absent in the non-quasifree meson production which has larger contributions from the nuclear volume.

It has to be concluded that the medium modifications leading to the depletion of cross section in nuclear photoabsorption are a subtle interplay of effects. Their investigation and the rigorous comparison to theoretical models requires the detailed study of differential cross sections and a deeper understanding of meson production in the nuclear medium. 


\section{REFERENCES}

1. K. Hagiwara, et al., Phys. Rev. D66 (2002) 010001.

2. K. Buechler, et al., Nucl. Phys. A570 (1994) 580-598.

3. M. MacCormick, et al., Phys. Rev. C53 (1996) 41-49.

4. F. Härter, et al., Phys. Lett. B401 (1997) 229-233.

5. A. Braghieri, et al., Phys. Lett. B363 (1995) 46-50.

6. J. Wißkirchen, PhD thesis, University of Bonn (1999) BONN-IR-99-21.

7. W. Langgärtner, et al., Phys. Rev. Lett. 87 (2001) 052001.

8. M. Kotulla, $\mathrm{PhD}$ thesis, University of Giessen.

9. E. Hourany, AIP Conference Proceedings 610 (INPC 2001) 362.

10. B. Krusche, et al., Phys. Rev. Lett. 74 (1995) 3736-3739.

11. F. Renard, et al., Phys. Lett. B528 (2002) 215-220.

12. J. A. Gomez Tejedor, E. Oset, Nucl. Phys. A600 (1996) 413-435.

13. J. C. Nacher, E. Oset, M. J. Vicente, L. Roca, Nucl. Phys. A695 (2001) 295-327.

14. B. Krusche, S. Schadmand, Prog. Part. Nucl. Phys. 51 (2003) 399-485.

15. A. Zabrodin, et al., Phys. Rev. C55 (1997) 1617-1620.

16. M. Wolf, et al., Eur. Phys. J. A9 (2000) 5-8.

17. V. Muccifora, et al., Phys. Rev. C60 (1999) 064616.

18. J. Arends, et al., Z. Phys. A305 (1982) 205.

19. B. Krusche, et al., Phys. Rev. Lett. 86 (2001) 4764-4767.

20. M. Roebig-Landau, et al., Phys. Lett. B373 (1996) 45-50.

21. H. Yamazaki, et al., Nucl. Phys. A670 (2000) 202-205.

22. S. Janssen, PhD thesis, University of Giessen (2002) and to be published.

23. U. Mosel, Prog. Part. Nucl. Phys. 42 (1999) 163-176.

24. J. Lehr, M. Effenberger, U. Mosel, Nucl. Phys. A671 (2000) 503-531.

25. M. Effenberger, A. Hombach, S. Teis, U. Mosel, Nucl. Phys. A614 (1997) 501-520.

26. M. Hirata, N. Katagiri, K. Ochi, T. Takaki, Phys. Rev. C66 (2002) 014612.

27. N. Bianchi, E. De Sanctis, M. Mirazita, V. Muccifora, Phys. Rev. C60 (1999) 064617.

28. M. Post, S. Leupold, U. Mosel, nucl-th/0309085.

29. T. Inoue, E. Oset, Nucl. Phys. A710 (2002) 354-370.

30. R. C. Carrasco, Phys. Rev. C48 (1993) 2333-2339.

31. J. Lehr, M. Post, U. Mosel, Phys. Rev. C68 (2003) 044601.

32. J. Lehr, U. Mosel, Phys. Rev. C64 (2001) 042202.

33. V. Kleber, et al., Eur. Phys. J. A9 (2000) 1-4.

34. J. G. Messchendorp, et al., Phys. Rev. Lett. 89 (2002) 222302.

35. B. Krusche, et al., Eur. Phys. J. A22 (2004) 347-351.

36. R. C. Carrasco, E. Oset, Nucl. Phys. A536 (1992) 445-508. 